\title{
Overlap of IgG4-related Sclerosing Cholangitis and Primary Biliary Cirrhosis
}

\author{
Reika Takemoto ${ }^{1}$, Yasuhiro Miyake ${ }^{2}$, Kenichi Harada $^{3}$, Yasuni Nakanuma ${ }^{3}$, Akio Moriya ${ }^{1}$, \\ Masaharu Ando ${ }^{1}$, Mamoru Hirohata ${ }^{1}$ and Kazuhide Yamamoto ${ }^{2}$
}

\begin{abstract}
We herein present a case of an overlap of immunoglobulin G4-related sclerosing cholangitis (IgG4-SC) and primary biliary cirrhosis (PBC). A 61-year-old man was diagnosed with PBC due to abnormal liver biochemical tests and positivity for serum anti-M2 antibody. However, his response to bezafibrate and ursodeoxycholic acid was insufficient. Five years later, his serum IgG4 level was found to increase. His liver biopsy specimens showed features of nonsuppurative destructive cholangitis in some portal tracts and periductal fibrosis with dense infiltration of IgG4-positive cells in other portal tracts. This case demonstrates that the serum IgG4 level may be worth measuring in patients with PBC refractory to conventional treatment.
\end{abstract}

Key words: autoimmune liver disease, immunoglobulin G4-related sclerosing cholangitis, overlap, primary biliary cirrhosis

(Intern Med 53: 1429-1433, 2014)

(DOI: 10.2169/internalmedicine.53.2250)

\section{Introduction}

Immunoglobulin G4-related disease (IgG4-RD) is a newly recognized fibro-inflammatory condition characterized by a dense lymphoplasmacytic infiltrate rich in IgG4-positive plasma cells, storiform fibrosis, and elevated serum IgG4 levels (1). IgG4-related sclerosing cholangitis (IgG4-SC) is classified as a biliary manifestation of IgG4-RD, and many cases respond well to corticosteroid treatment $(2,3)$.

Primary biliary cirrhosis (PBC) is a chronic cholestatic disease with a progressive course characterized by the presence of serum anti-mitochondrial antibodies and histological nonsuppurative destructive cholangitis; it is generally treated with ursodeoxycholic acid (UDCA) (4). For PBC patients showing insufficient response to UDCA, combination therapy of bezafibrate and UDCA is usually effective (5).

IgG4-SC and PBC are two distinct autoimmune liver diseases. However, until now, just two cases of an overlap of IgG4-SC and PBC have been reported $(6,7)$, and both also had autoimmune pancreatitis (AIP). Among IgG4-SC pa- tients, $90 \%$ have AIP, so the presence of AIP helps the diagnosis of IgG4-SC (3). We herein report a case of an overlap of IgG4-SC and PBC without the concurrence of AIP.

\section{Case Report}

A 61-year-old man presented to the hospital for evaluation of abnormal liver biochemical tests with preferential elevations of serum alkaline phosphatase (ALP) and gammaglutamyltranspeptidase (GGT) levels: ALP, 2,075 IU/L; GGT, 1,433 IU/L; aspartate aminotransferase (AST), 86 IU/ $\mathrm{L}$, alanine aminotransferase (ALT), $105 \mathrm{IU} / \mathrm{L}$; and total bilirubin, $1.9 \mathrm{mg} / \mathrm{dL}$ (Table). Serum levels of IgG and IgM were $1,976 \mathrm{mg} / \mathrm{dL}$ and $945 \mathrm{mg} / \mathrm{dL}$, respectively. Anti-M2 antibody serum level was increased to $79 \mathrm{U} / \mathrm{mL}$. There was no history of alcohol abuse, blood transfusion, or hepatotoxic medication. Negative serology excluded viral hepatitis. Enhanced computed tomography (CT) showed no intrahepatic mass, and magnetic resonance cholangiopancreatography (MRCP) did not show common bile duct or hepatic duct stricture (Fig. 1). He was diagnosed with

\footnotetext{
${ }^{1}$ Department of Internal Medicine, Mitoyo General Hospital, Japan, ${ }^{2}$ Department of Gastroenterology and Hepatology, Okayama University Graduate School of Medicine, Dentistry, and Pharmaceutical Sciences, Japan and ${ }^{3}$ Department of Human Pathology, Kanazawa University Graduate School of Medicine, Japan

Received for publication December 4, 2013; Accepted for publication January 26, 2014

Correspondence to Dr. Yasuhiro Miyake, miyake43@md.okayama-u.ac.jp
} 
Table. Laboratory Data at First Visit and at Liver Biopsy

\begin{tabular}{lll}
\hline Variable & At first visit & At liver biopsy \\
\hline White blood cell count $\left(3,500-9,000 / \mathrm{mm}^{3}\right)$ & 4,350 & 5,840 \\
Hemoglobin $(13.5-17.0 \mathrm{~g} / \mathrm{dL})$ & 13.1 & 12.1 \\
Platelet count $\left(13.0-35.0 \times 10^{4} / \mathrm{mm}^{3}\right)$ & 23.7 & 19.8 \\
Albumin $(3.5-5.5 \mathrm{~g} / \mathrm{dL})$ & 3.3 & 3.0 \\
Total bilirubin $(0.2-1.0 \mathrm{mg} / \mathrm{dL})$ & 1.9 & 2.2 \\
Aspartate aminotransferase $(10-40 \mathrm{IU} / \mathrm{L})$ & 86 & 77 \\
Alanine aminotransferase $(4-45 \mathrm{IU} / \mathrm{L})$ & 105 & 58 \\
Alkaline phosphatase $(105-340 \mathrm{IU} / \mathrm{L})$ & 2,075 & 831 \\
Gamma-glutamyltranspeptidase $(5-70 \mathrm{IU} / \mathrm{L})$ & 1,433 & 793 \\
Amylase $(37-120 \mathrm{IU} / \mathrm{L})$ & 66 & 87 \\
Lipase $(11-53 \mathrm{IU} / \mathrm{L})$ & $\mathrm{NT}$ & 48 \\
Immunoglobulin G $(850-1,820 \mathrm{mg} / \mathrm{dL})$ & 1,976 & 2,229 \\
Immunoglobulin G4 $(4.8-105 \mathrm{mg} / \mathrm{dL})$ & $\mathrm{NT}$ & 555 \\
Immunoglobulin A $(105-390 \mathrm{mg} / \mathrm{dL})$ & 598 & 691 \\
Immunoglobulin M $(40-200 \mathrm{mg} / \mathrm{dL})$ & 945 & 472 \\
Anti-nuclear antibody $(<1: 40)$ & $<1: 40$ & $1: 80$ \\
Anti-M2 antibody $(<7 \mathrm{U} / \mathrm{mL})$ & 79 & 90 \\
Anti-myeloperoxidase Anti-neutrophil cytoplasmic antibody $(<10 \mathrm{EU})$ & $\mathrm{NT}$ & $<10$ \\
Thyroid stimulating hormone $(0.35-5.00 \mu \mathrm{IU} / \mathrm{mL})$ & $\mathrm{NT}$ & 1.59 \\
Free-T4 $(0.7-1.5$ ng/dL) & $\mathrm{NT}$ & 1.4 \\
Anti-thyroid peroxidase antibody $(<1: 100)$ & $\mathrm{NT}$ & $<1: 100$ \\
Anti-thyroglobulin antibody $(<1: 100)$ & $\mathrm{NT}$ & $<1: 100$ \\
\hline NT: & & \\
\hline
\end{tabular}

NT: not tested.

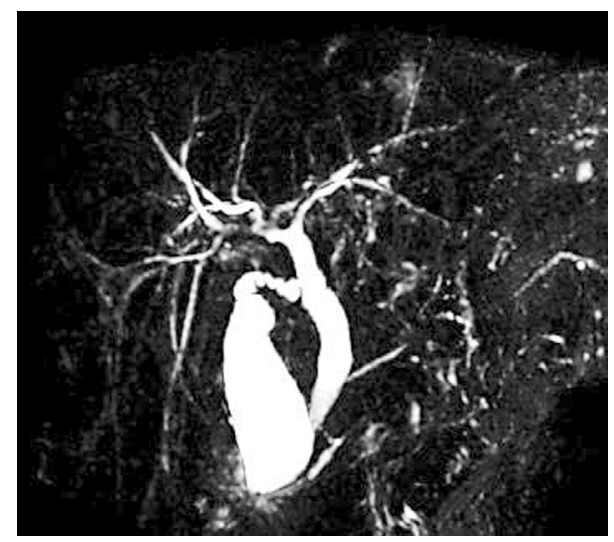

Figure 1. MRCP does not show common bile duct or hepatic duct stricture.

PBC (4), and oral UDCA $600 \mathrm{mg}$ daily was introduced. Seven months later, his liver biochemical tests improved to some extent but remained abnormal: AST, 56 IU/L; ALT, 54 IU/L; ALP, 1,002 IU/L; GGT, 495 IU/L; and total bilirubin, $0.8 \mathrm{mg} / \mathrm{dL}$. Therefore, oral bezafibrate $400 \mathrm{mg}$ daily was added (5).

Five years after the introduction of UDCA treatment, his liver biochemical tests remained abnormal: AST, 74 IU/L; ALT, 56 IU/L; ALP, 692 IU/L; GGT, 665 IU/L; and total bilirubin, $2.0 \mathrm{mg} / \mathrm{dL}$. Serum IgM level decreased to $532 \mathrm{mg} /$ $\mathrm{dL}$ although serum IgG level slightly increased to $2,358 \mathrm{mg}$ / dL. Serum anti-nuclear antibody was positive, with a titer of 1:80. Serum IgG4 level increased to $449 \mathrm{mg} / \mathrm{dL}$. His MRCP showed bile duct stricture in the hilar hepatic lesions but no features of AIP (Fig. 2). The serum levels of amylase and lipase were within normal limits. Enhanced CT showed no intra-hepatic mass. Endoscopic retrograde cholangiopancreatography was not performed because the patient's informed

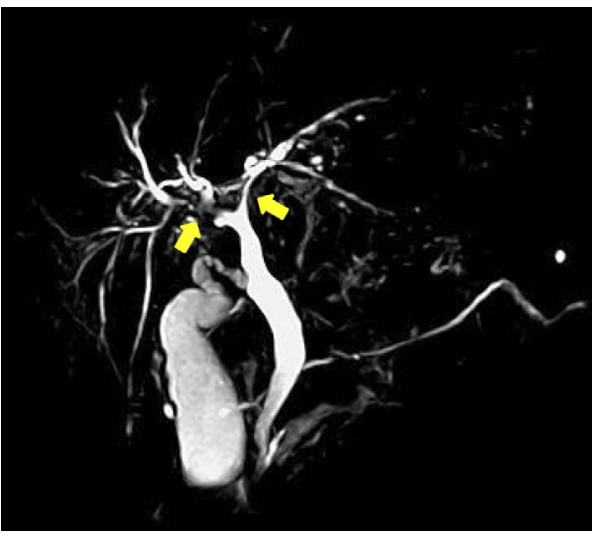

Figure 2. MRCP shows bile duct stricture in the hilar hepatic lesions (yellow arrows) without any features of autoimmune pancreatitis.

consent could not be obtained. In order to diagnose his liver disease, liver biopsy specimens were taken under ultrasonography, based on his informed consent. His liver biopsy specimens revealed ductular proliferation, interface hepatitis with marked portal lymphoplasmacytic infiltration, and fibrosis arranged focally in a storiform pattern, but obliterative phlebitis features were not observed (Fig. 3A). Features of nonsuppurative destructive cholangitis were noted in some portal tracts (Fig. 3B), and periductal fibrosis with lymphoplasmacytic infiltration was observed in other tracts (Fig. 3C). IgG4 immunostaining revealed more than 30 IgG4-positive cells per high-power field (Fig. 3D). He was diagnosed with an overlap of $\operatorname{IgG} 4-\mathrm{SC}$ and $\operatorname{PBC}(4,8)$. Laboratory data at liver biopsy are shown in Table. Using the revised scoring system proposed by the International Autoimmune Hepatitis Group (9), the score was 6.

Thereafter, corticosteroid treatment was introduced with 

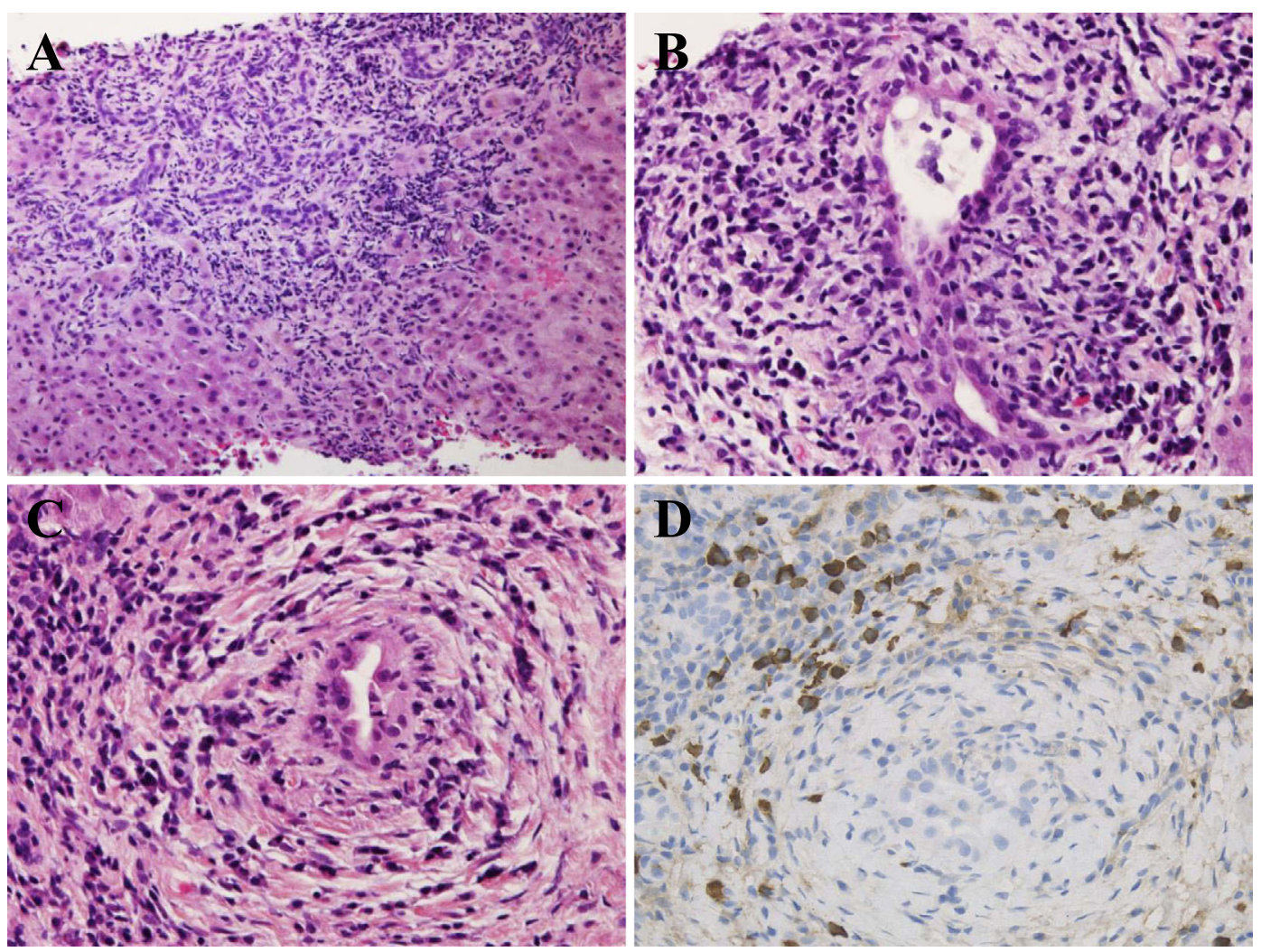

Figure 3. Pathological findings of a liver biopsy. (A) Ductular proliferation, interface hepatitis with marked portal lymphoplasmacytic infiltration, and fibrosis arranged focally in a storiform pattern. (B) Nonnsuppurative destructive cholangitis. (C) Periductal fibrosis with lymphoplasmacytic infiltration. (D) IgG4 immunostaining revealed more than 30 IgG4-positive cells per high-power field. (C) and (D) are from the same area of serial sections.

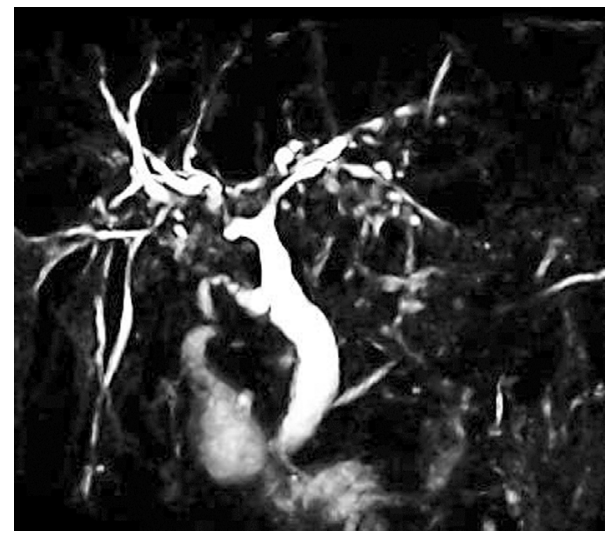

Figure 4. MRCP after 24-weeks corticosteroid treatment seems to show some improvement of bile duct stricture in the hilar hepatic lesions.

oral prednisolone $30 \mathrm{mg}$ daily, tapered by $5 \mathrm{mg}$ weekly to $10 \mathrm{mg}$ daily. Twenty-four weeks after the introduction of corticosteroid treatment, his liver biochemical tests did not normalize. However, serum IgG4 level decreased to $173 \mathrm{mg}$ / $\mathrm{dL}$, and his MRCP seemed to show some improvement of bile duct stricture in the hilar hepatic lesions (Fig. 4).

\section{Discussion}

Positivity for serum anti-M2 antibody has a high sensitivity and specificity for the diagnosis of PBC (10). In addition, the histological features of nonsuppurative destructive cholangitis in the present case were compatible with PBC (4). On the other hand, elevated serum IgG4 level and histological features of periductal fibrosis with dense infiltration of IgG4-positive cells met the diagnostic criteria for IgG4-SC (8). However, the differential diagnosis of IgG4$\mathrm{SC}$ from primary sclerosing cholangitis (PSC) is difficult in some cases. Cholangiographic findings are usually useful for the discrimination of IgG4-SC from PSC. The typical cholangiographic findings of IgG4-SC are segmental strictures, long strictures with pre-stenotic dilation, and strictures of the lower common bile duct; however, band-like strictures, a beaded or "pruned-tree" appearance and diverticulum-like out-pouching are significantly more common in PSC (8). The MRCP findings of the present case were classified as Type $2 b$ according to the cholangiographic classification of IgG4-SC (8). Furthermore, serum IgG4 levels above 540 $\mathrm{mg} / \mathrm{dL}$ have been reported to have a very high specificity for the diagnosis of IgG4-SC (>99\%) (11). In the present case, the serum IgG4 level at the time of liver biopsy was $555 \mathrm{mg} / \mathrm{dL}$. Therefore, the present case was diagnosed with 
an overlap of IgG4-SC and PBC.

Recently, IgG4-associated autoimmune hepatitis (AIH) has been reported to be found in over 3\% of classical AIH cases in a Japanese cohort; it is characterized by high serum IgG4 and IgE levels and IgG4-bearing plasma cell infiltration in the liver (12). However, IgG4-associated AIH is diagnosed based on the revised scoring system proposed by the International Autoimmune Hepatitis Group (9). The present case did not meet the criteria for AIH.

The effectiveness of corticosteroid treatment is one of the clinical characteristics in IgG4-SC (8). However, the present case showed an incomplete response to corticosteroid therapy. A complete response is only achieved in $60 \%$ of IgG4SC patients, although $97 \%$ show some response (3). In addition, advanced-stage IgG4-SC has been suggested to be sometimes unresponsive to corticosteroid therapy because cases of IgG4-SC show a predominantly inflammatory nature at the early stage, followed by relatively less inflammation, but marked fibrous scarring later (13). In the present case, more than 5 years had passed between the first visit and the introduction of corticosteroid therapy. Therefore, an insufficient response to corticosteroid therapy does not negate the diagnosis of IgG4-SC in the present case.

Pancreatic involvement in PBC has been previously reported. Abnormal features of pancreatic ducts by endoscopic retrograde pancreatography are observed in approximately $10-20 \%$ of $\mathrm{PBC}$ patients $(14,15)$. Considering that two cases of an overlap of IgG4-SC and PBC with concurrent AIP have been reported $(6,7)$, some PBC patients may have AIP. In addition, some PBC patients have been reported to have idiopathic retroperitoneal fibrosis (16-18), which is one of the manifestations of IgG4-RD (2). Therefore, a small subset of PBC patients may have IgG4-RD. However, clinical features of an overlap of IgG4-RD and PBC remain unknown because of its rarity. All five PBC patients with AIP or idiopathic retroperitoneal fibrosis previously reported were male $(6,7,16-18)$. The proportions of women are $87 \%$ and $17-38 \%$ in PBC and IgG4-RD, respectively $(1,19)$, thus suggesting that gender may be associated with the occurrence of IgG4-RD in PBC. Hereafter, a nationwide survey of an overlap between IgG4-RD and PBC will be needed to elucidate the clinical features.

Are there common features in PBC and IgG4-RD pathogeneses? The role of bacteria in PBC development is well known. There is molecular mimicry between the human E2 component of the pyruvate dehydrogenase complex (target antigen of anti-M2 antibody) and proteins of Escherichia coli and serum anti-M2 antibodies in PBC patients bind to E. coli proteins (20). Furthermore, Toll-like receptor 4 (TLR4) ligand-stimulated natural killer cells destroy autologous biliary epithelial cells in the presence of interferon alpha synthesized by TLR3 ligand-stimulated monocytes (21). On the other hand, stimulation with TLR3 and TLR4 ligands enhances $\mathrm{IgG} 4$ production by peripheral blood mononuclear cells in IgG4-RD patients (22). Therefore, an excessive innate immune response to microbes seems to be associated with the pathogeneses of PBC and IgG4-RD.

We herein described a case of an overlap of IgG4-SC and PBC without concomitant AIP. This case demonstrates that the serum IgG4 level may be worth measuring in patients with $\mathrm{PBC}$ refractory to conventional treatment.

The authors state that they have no Conflict of Interest (COI).

\section{References}

1. Stone JH, Zen Y, Deshpande V. IgG4-related disease. N Engl J Med 366: 539-551, 2012.

2. Okazaki K, Uchida K, Ikeura T, Takaoka M. Current concept and diagnosis of IgG4-related disease in the hepato-bilio-pancreatic system. J Gastroenterol 48: 303-314, 2013.

3. Ghazale A, Chari ST, Zhang L, et al. Immunoglobulin G4associated cholangitis: clinical profile and response to therapy. Gastroenterology 134: 706-715, 2008.

4. Lindor KD, Gershwin ME, Poupon R, Kaplan M, Bergasa NV, Heathcote EJ; American Association for Study of Liver Diseases. Primary biliary cirrhosis. Hepatology 50: 291-308, 2009.

5. Honda A, Ikegami T, Nakamuta M, et al. Anticholestatic effects of bezafibrate in patients with primary biliary cirrhosis treated with ursodeoxycholic acid. Hepatology 57: 1931-1941, 2013.

6. Naitoh I, Nakazawa T, Hayashi K, et al. A case of IgG4-related sclerosing cholangitis overlapped with primary biliary cirrhosis. Intern Med 51: 1695-1699, 2013.

7. Wiegand J, Neid M, Kaiser T, et al. Coexistence of autoimmune pancreatitis and primary biliary cirrhosis in a Caucasian patient: a rare cause of cholestasis. Z Gastroenterol 44: 1227-1229, 2006.

8. Ohara H, Okazaki K, Tsubouchi H, et al. Research Committee of IgG4-related Diseases; Research Committee of Intractable Diseases of Liver and Biliary Tract; Ministry of Health, Labor and Welfare, Japan; Japan Biliary Association. Clinical diagnostic criteria of IgG4-related sclerosing cholangitis 2012. J Hepatobiliary Pancreat Sci 19: 536-542, 2012.

9. Alvarez F, Berg PA, Bianchi FB, et al. International Autoimmune Hepatitis Group Report: review of criteria for diagnosis of autoimmune hepatitis. J Hepatol 31: 929-938, 1999.

10. Berg PA, Klein R, Lindenborn-Fotinos J, Klöppel W. ATPaseassociated antigen (M2): marker antigen for serological diagnosis of primary biliary cirrhosis. Lancet 2: 1423-1426, 1982.

11. Ohara $H$, Nakazawa $T$, Kawa $S$, et al. Establishment of a serum IgG4 cut-off value for the differential diagnosis of IgG4-related sclerosing cholangitis: a Japanese cohort. J Gastroenterol Hepatol 28: 1247-1251, 2013.

12. Umemura $\mathrm{T}$, Zen $\mathrm{Y}$, Hamano $\mathrm{H}$, et al. Clinical significance of immunoglobulin G4-associated autoimmune hepatitis. J Gastroenterol 46 (Suppl 1): 48-55, 2011.

13. Nakazawa T, Naitoh I, Hayashi K, Miyabe K, Simizu S, Joh T. Diagnosis of IgG4-related sclerosing cholangitis. World J Gastroenterol 19: 7661-7670, 2013.

14. Nishimori I, Morita M, Kino J, et al. Pancreatic involvement in patients with Sjögren's syndrome and primary biliary cirrhosis. Int J Pancreatol 17: 47-54, 1995.

15. Fausa O, Kolmannskog F, Ritland S. The pancreatic ducts in primary biliary cirrhosis and sclerosing cholangitis. Scand J Gastroenterol Suppl 107: 32-35, 1985.

16. Tang KH, Schofield JB, Powell-Jackson PR. Primary biliary cirrhosis and idiopathic retroperitoneal fibrosis: a rare association. Eur J Gastroenterol Hepatol 14: 783-786, 2002.

17. Shikuwa S, Omagari K, Mizuta Y, et al. Primary biliary cirrhosis associated with idiopathic retroperitoneal fibrosis. J Gastroenterol 35: 646-648, 2000. 
18. Sevenet F, Capron-Chivrac D, Delcenserie R, Lelarge C, Delamarre J, Capron JP. Idiopathic retroperitoneal fibrosis and primary biliary cirrhosis. A new association? Arch Intern Med 145: 21242125, 1985.

19. Harada K, Hirohara J, Ueno Y, et al. Incidence of and risk factors for hepatocellular carcinoma in primary biliary cirrhosis: national data from Japan. Hepatology 57: 1942-1949, 2013.

20. Bogdanos DP, Baum H, Grasso A, et al. Microbial mimics are major targets of crossreactivity with human pyruvate dehydroge- nase in primary biliary cirrhosis. J Hepatol 40: 31-39, 2004.

21. Shimoda S, Harada K, Niiro H, et al. Interaction between Toll-like receptors and natural killer cells in the destruction of bile ducts in primary biliary cirrhosis. Hepatology 53: 1270-1281, 2011.

22. Watanabe T, Yamashita K, Fujikawa S, et al. Involvement of activation of toll-like receptors and nucleotide-binding oligomerization domain-like receptors in enhanced IgG4 responses in autoimmune pancreatitis. Arthritis Rheum 64: 914-924, 2012.

(C) 2014 The Japanese Society of Internal Medicine http://www.naika.or.jp/imonline/index.html 